\title{
Prevalence of HIV in a Cohort of Pregnant Women Attending a Tertiary Hospital in Ibadan, Nigeria
}

\author{
Iheanyi O. Okonko*, Anwuli U. Osadebe, Okechukwu Onianwa, Stella Okereke
}

Department of Microbiology, University of Port Harcourt, Nigeria

Copyright $(2019$ by authors, all rights reserved. Authors agree that this article remains permanently open access under the terms of the Creative Commons Attribution License 4.0 International License

\begin{abstract}
HIV/AIDS is one of the major barriers to community development. It is a global pandemic with high prevalence rates worldwide. This research was carried out to determine the prevalence of HIV among pregnant women attending the antenatal clinic at the University College Hospital, Ibadan using Enzyme Linked Immunosorbent Assay (ELISA) Gen Screen ${ }^{\mathrm{TM}}$ ULTRA HIV Ag-Ab Kit. HIV prevalence was found to be $26.4 \%$. A total of 91 samples were tested, $24(26.4 \%)$ were found to be positive and $67(73.6 \%)$ negative. Women between the ages $25-31$ had the highest prevalence rate of $16.5 \%$ while those between the ages of $39-45$ years had the least prevalence rate of $1.1 \%$. The results revealed a relatively high prevalence rate for HIV among pregnant women which suggests the need for active surveillance and implementation of preventive measures to avoid further spread of the virus. Pregnant women need to be continually tested for HIV and those infected monitored closely to prevent mother to child transmission.
\end{abstract}

Keywords Antenatal, Epidemiology, HIV/AIDS, Mother-to-child Transmission, Pregnant Women, Prevalence

\section{Introduction}

HIV is a slow replicating virus (lentivirus) that causes Acquired Immune Deficiency Syndrome (AIDS), a condition leading to gradual collapse of the human immune system allowing invasion by lethal opportunistic pathogens and cancers [1]. HIV infection leads to low levels of CD4+ T-cells. Infection with HIV occurs via contact with infected blood, semen, vaginal fluid, pre-ejaculate or breast milk. People who have become infected with HIV may be asymptomatic for up to ten years though they remain able to infect others.

Symptoms related to HIV are usually due to the different opportunistic infections in the body. These symptoms range from diarrhoea, fatigue, fever, frequent vaginal yeast infections and headaches to seborrheic dermatitis, psoriasis, thrush, muscle stiffness and numbness [2, 3].

HIV/ AIDS has claimed more than 35 million lives since its characterisation in 1981 [4]. As at 2017, the number of individuals estimated to be living with HIV/ AIDS worldwide was 31.1 million - 43.9 million counting 1.8 million new infections in that same year. Adults account for $86 \%$ of this figure. There are 19.6 million (53.1\%) people living with HIV/AIDS (PLWHIV) in eastern and southern Africa and 6.1 million (16.5\%) in western and central Africa. Approximately 670,000 - 1.3 million deaths from AIDS - related illnesses were recorded across the globe in 2017. Prevalence in Nigeria has fluctuated over the last few decades; from lows of $1.8 \%$ in 1991 through $5.8 \%$ and $3.0 \%$ in 2001 and 2014 respectively to $2.9 \%$ in $2016[5,6]$. Nigeria has the second largest HIV epidemic in the world. The country currently accounts for 3.2 million PLWHIV with an average of 160,000 AIDS-related deaths annually. Of the estimated 220,000 new infections in Nigeria, 37,000 were from mother-to-child transmission. As at 2017, Rivers state, Nigeria recorded an HIV prevalence of $4.1-6.0 \%[6,7,8]$. With increasing access to antiretroviral therapy (ART), there has been a gradual decline in the occurrence of HIV/AIDS - related deaths even though the number of new infections amongst the poorly educated and low income group continues to rise in certain countries and has plateaued in others. The prevalence of fresh HIV infection is thus, persistently high in several countries across the sub-Sahara with South Africa (23\%), Nigeria (15\%), Uganda (10\%), Mozambique $(8 \%)$ and Kenya $(7 \%)$ ranked top $[6,9,10]$. HIV is considered a major concern in the achievement of the Sustainable Development Goals (SDGs) with particular emphasis on the $3^{\text {rd }}$ goal of healthy living and the promotion of well-being for all including the pledge to terminate the scourge of infectious diseases and eliminate epidemics across the globe such as those of HIV/ AIDS and tuberculosis by 2030 [11].

Poverty, gender-based vulnerability and low risk perception are thought to be the key drivers of the HIV pandemic [12]. Most studies have highlighted heterosexual 
intercourse as the main route of transmission in Nigeria being responsible for $80 \%$ of incidents, other studies found that homosexual intercourse and intravenous drug use are equally important means of spreading HIV. The relevant high risk groups including commercial sex workers, prison inmates and drug addicts are thought to make up $1 \%$ of the Nigerian population [4, 13]. HIV/ AIDS is the major reason for mortality amongst women of child-bearing age; it further plays a key role in the mortality among children and infants as well. Studies have shown that the HIV-infected expectant mothers have 8 times greater mortality rates than found in uninfected pregnant women. Furthermore, about $24 \%$ of maternal mortality in the sub-Sahara has been attributed to HIV/ AIDS. The HIV-related maternal deaths range from $7 \%-21 \%$ globally with higher values in the sub-Saharan region. [11, 14, 15]. It is opined that absent well-tailored management strategies, Mother to Child Transmission (MTCT) of HIV has been known to rise to highs of $25 \%-40 \%$ prevalence. The risk of MTCT in infected breast-feeding women is $25 \%-45 \%$ and $15-25 \%$ in mothers that do not breastfeed [12]. In most developed countries, incidence of MTCT have fallen below $2 \%$ with the employment of effective interventions.

Prevalence studies are fundamental to combatting the HIV/AIDS pandemic and achieving the Sustainable Development Goals (SDGs) in the sub-Saharan region. Antenatal clinics have served as the prime source of data for determining both regional and national incidence and prevalence of HIV especially in countries with widespread HIV epidemics such as found in the sub-Sahara. Such statistics are valuable in epidemiological monitoring as HIV prevalence trends in antenatal clinics will often mirror the trends in the general population [16, 17]. This study, therefore, aimed to establish the prevalence of HIV among pregnant women attending the antenatal clinic at the University College Hospital, Ibadan, Nigeria. The objectives were to determine age-specific trends in prevalence among the participants and to ascertain prevalence statistics within age groups.

\section{Materials and Methods}

\subsection{Study Design and Study Population}

The study was an institution-based transverse study conducted at the University College Hospital (UCH), Ibadan, Nigeria. The UCH is a 1000-bed hospital attached to the University of Ibadan and serves the western region of Nigeria. The hospital runs about 96 consultative out-patient clinics including one for people living with HIV/AIDS.

The study population comprised ninety-one (91) pregnant women between the ages of 18 and 45 years attending the antenatal clinic at the UCH. The sample size was determined using single population proportion formula working under the assumed working proportion of $6.5 \%$ with $5 \%$ tolerated margin of sampling error at $95 \%$ confidence interval.

\subsection{Inclusion Criteria and Data Collection}

Only pregnant women attending the antenatal clinic who voluntarily consented to participation were included in the study. Participants had to be confirmed pregnant. Non-pregnant women and those unwilling to provide informed consent were considered ineligible. Participants' details were collected via structured interviews.

\subsection{Ethical Considerations}

The study was carried out according to ethical medical research standards for human subjects. Ethical approval for the study was given by hospital management. The purpose, importance and method of the study was explained to the attendees in detail both in English and in the local language before requesting consent for participation. Participants were further informed of their right to withdraw consent at any point during the study. All personal information given was handled anonymously. All the participants signed consent forms and filled out questionnaires anonymously with their medical history and demographic details

\subsection{Assay for HIV Antibodies}

Approximately $5 \mathrm{ml}$ of venous blood samples were collected using sterile syringes and transferred into anticoagulant bottles. The bottles were sent to the medical laboratory for screening in a collection bode. The blood samples were separated into their components by centrifuging at 2000 resolution per minutes (rpm) for five minutes to obtain sera. Following separation, the obtained serum was collected and transferred into sterile labelled serum bottles and stored at $4^{\circ} \mathrm{C}$ until use. Screening for HIV antibodies was done using Enzyme Linked Immunosorbent Assay (ELISA) Gen Screen ${ }^{\text {TM }}$ ULTRA HIV Ag-Ab Kit. The test and interpretation of the results were carried out according to the kit manufacturer's specifications. Samples found to be positive were re-tested for confirmation.

\subsection{Statistical Analyses}

The generated data were presented in descriptive statistics. Statistical Package for the Social Sciences $(\mathrm{SPSS})^{\circledR} \quad 21.0 \quad$ International Business Machines $^{\circledR}$ Corporation (IBM), NY, USA) and Microsoft Excel 2017 were used to analyse the data. Statistical significance of data sets was determined at $p \leq 0.05$. Prevalence was determined by comparing the number of pregnant women positive for HIV antibodies with the total number tested. 


\section{Results and Discussion}

The HIV prevalence was $26.4 \%$ with 24 out of the 91 pregnant women testing positive. As highlighted in Figures 1 and 2, the greatest number of HIV positive individuals were found in the $25-31$ years age range followed by 18 24 years, $32-38$ years and then $39-45$ year age ranges with a $37.5 \%$ occurrence within the $25-31$ age group. Prevalence among these groups were $16.48 \%, 5.49 \%$, $3.29 \%$ and $1.10 \%$ respectively. An assessment of occurrence within the age groups revealed that age groups $25-31$ years and $18-24$ years still dominated. Statistical analyses underscored a significant difference, at $p<0.05$, between HIV seropositivity and age of pregnant women. Generally, there were significant differences in HIV seropositivity between the different age groups at 0.05 confidence interval.

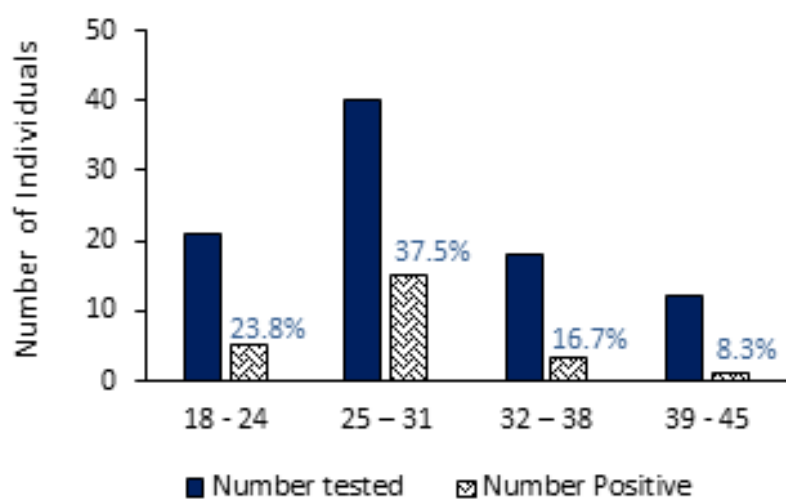

Figure 1. Occurrence of HIV among the Participants indicating Incidence within Groups

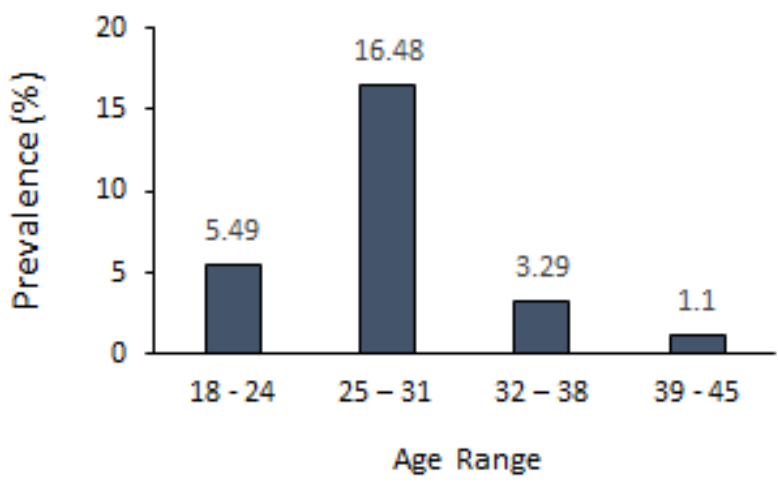

Figure 2. Prevalence of HIV according to the Age of Participants

The somewhat exceptionally high prevalence of HIV in this study is to be expected as the individuals investigated complained of ill-health; furthermore, numerous reports attest to the feminisation of the HIV epidemic particularly in the sub-Sahara. A study on women would therefore yield somewhat elevated values as women generally have higher prevalence rates than males. This higher prevalence in females is buttressed by results from similar studies [18, 19]. Oleribe et al. [4] surmised that though males in the region are more sexually active and more willing to test for
HIV, they still have much lower incidence rates than females. The $26.4 \%$ prevalence in this study is much higher than found in similar studies. Eaton et al. [17] recorded a $1.4 \%$ drop in HIV prevalence in West Africa from $4.3 \%$ to $2.9 \%$ overall. These values are much lower than found in this study but somewhat comparable to other similar studies. A study in Port Harcourt, Rivers state, Nigeria found a prevalence of $3.0 \%$ among expectant mothers attending antenatal clinic [14] while another study of 216 antenatal clinic participants in Jos, Plateau state, Nigeria observed a prevalence of $8.3 \%$ [12]. A meta-analysis of 8443 women attending antenatal clinics in Abuja, Nigeria placed prevalence rates at $11.5 \%$ [16]. With expectant mothers in Cameroon, a 13.1\% HIV prevalence was found [15]. A study in Lusaka, however, found a similar HIV prevalence of $26 \%$ among pregnant women in Lusaka, Zambia with some of the provinces studied having values of up to $28.9 \%$ [11]. Several reasons could be advanced for the exceptionally high prevalence in the current study. One such reason is poorly targeted intervention activities. The high prevalence could be indicative of the inadequacy of interventions to meet the complex social and reproductive health needs of women in the region. There is also the issue of polygamy and multiple sex partners or unprotected sex with partners that are unfaithful.

Statistics from a number of studies have highlighted age of participants as the most crucial factor in epidemiological studies. Variations in incidence from one age group to the other reflects the involvement of age in determining prevalence rates. Global statistics reveal that $15 \%$ of HIV positive females fall within the $15-24$ years group. This group bear a disproportionate burden of infection having higher prevalence than their male counterparts [20]. Furthermore, they are thought to develop infection five (5) to seven (7) years before males in the same age bracket [10]. Other researchers opined that HIV prevalence was higher for females in all age groups except the $40-44$ years group [13]. It has been found that in Africa, girls aged 15-24 years are two times more likely to be infected by HIV than any other age group. Moreover, 3 out of every 4 newly infected individuals in Africa are young women aged between 15 and 19 years old [6]. This is especially true of eastern and southern Africa.

The results in this study do not necessarily tally with the above global statistics as it was the pregnant women aged $25-31$ years that demonstrated the preponderance for HIV seropositivity. This group was however, followed by women in the $18-24$ years age group in accordance with global studies. Singh et al. [9] found that HIV positive female patients tended to have a mean age of 41.63 years while Sharma and Chauhan [21] underlined 25 - 34 years and $35-49$ years as the age groups with the greatest HIV prevalence. Other studies further highlight the dominance of females aged $20-29$ years [12, 14, 22, 23]. Observations from La'ah and Ayiwulu [24] and Fouedjio et al. [15] extended this predominance to the $20-34$ years age range. Analysis by Eaton et al. [17] revealed that HIV 
prevalence declined appreciably in females within the 1524 years age group but rose significantly among those aged $35-49$ years. One study maintains that there occurs an inverse trend with regards to maternal age and HIV seropositivity. They suggest that a higher incidence will often be observed in the $15-24$ years age group when compared to mothers aged $30-49$ years and older [16].

This current study reported a significant statistical relationship between maternal age and HIV positivity within the antenatal clinic which stands at variance with the conclusions of certain studies that reported the absence of any significant link between HIV- 1/2 seropositivity and age $[25,26]$. A surveillance study in Abuja, Nigeria also found that HIV seropositivity correlated with maternal age but was not influenced by parity [16]. One study implied that it was not uncommon to find HIV prevalence rates higher in hospital-based studies when compared with studies in the general population [12]. This may not necessarily apply to this study because the participants were not primarily at the hospital due to ill health. The findings in this study further contradict the observations by Eaton et al. [17] that trends in HIV prevalence within the sub-Sahara had moved towards less-fertile women while declining among younger mothers. They stated that prevalence among pregnant women in the region had shown a more precipitous decline than observed among women in general. The results from the current study seem to negate this.

Various factors contribute to the observed gender gap and distribution of HIV seropositivity among different age groups. These factors influence the high prevalence rate found in this study as well. Such factors include poverty, illiteracy, skewed gender-based norms in the African setting, child marriage and polygamy, harmful cultural practices, sexual violence towards women, disability, human right abuses and political influences [8, 27]. Women will often bear the brunt of sexual health issues because of biased cultural practices that deny women the right to make decisions about sex and marriage. Polygamy is rife; in these settings, wives will normally be infected by a HIV positive husband [9, 28, 29]. Prevalence rates are also connected with certain high risk behaviours such as not using a condom and sex for commercial purposes. This group tend to have a history of at least one sexually transmitted disease. The lack of awareness of the transmission and management of HIV and the stigmatisation of infected mothers by both the community and health workers has been highlighted as a key influencing factors as well $[14,27]$. The earlier infected persons can be put on antiretroviral therapy (ART), the better, however, the privacy of HIV positive individuals is often very poorly handled which discourages the concerned individuals from coming forward for proper sensitisation and treatment.

The high prevalence calls into question the effectiveness of HIV/AIDS management programmes in the region. The recognised challenges must be effectively tackled by government agencies at all levels and healthcare centres if a consistent decline in prevalence within the region is to be achieved. The need to educate young girls is evident from the prevalence bias noted in this study and other similar ones. Empowering women both psychologically and financially to take control of their bodies would play a vital part here. All the factors that promote the vulnerability of females to infection must be addressed. The high prevalence rate observed indicates an urgent need for a cogent HIV surveillance in antenatal clinics. Surveillance systems would have to spread in different directions to include smaller clinics in rural areas which will improve coverage of rural populations, the scaling up of HIV programmes such as prevention of mother-to-child transmission and voluntary testing and counselling which present both a challenge and an opportunity for improvement of current surveillance systems. Expectant mothers should be encouraged to know their HIV statuses. This would help minimise transmission to the unborn child and partners. Where a mother is found to be HIV positive, urgent intervention in form of antiretroviral therapy (ART) as outlined by World Health Organisation (WHO) regulations should commence to ensure the health of the mother and to prevent mother-to-child-transmission, MTCT. Constant surveillance is strongly recommended. Studies such as this are encouraged as knowledge of national, state and community-level prevalence rates would inform evidence-based decision making and policy formulation; a necessity for reigning in this crisis. Monitoring of infection among attendees of antenatal clinics provides avenues for adequate counselling by professionals, reinforces safe sex practices reducing the risk of infecting an HIV negative partner, decreases the incidence of HIV orphans and empowers the women allowing them to make informed decisions.

While investigating trends in a capital city play a role in monitoring the HIV epidemic, it should, however, be noted that the observed trends may not necessarily give a true indication of the problem and the effectiveness of management programmes as such programmes tend to already be centred around capital cities and large towns. Studies of trends in more rural locations may, therefore, prove to be more representative.

A key limitation of the study is that factors beyond pregnancy with the potential to impact on the representativeness of HIV prevalence trends amongst the antenatal clinic attendees were not explored. Such factors include the tendency of antenatal clinics in government hospitals to consist of mothers from low-income or more rural backgrounds as opposed to what is found in private clinics and the changing patterns in antenatal clinic attendance. Further research on the influence of these factors is encouraged. 


\section{Conclusions}

The study found a high prevalence of $26.4 \%$ HIV seropositivity among a group of women attending antenatal clinic at the University College Hospital, Ibadan, Nigeria. The findings reinforced the significant relationship between the age of the mothers and HIV positivity. Expectant mothers aged $25-31$ years were found to be more prone to HIV infection than other age groups. The high prevalence observed informs the urgent need for effective interventions at both national and state levels to prevent mother-to-child transmission of the virus. Further research to clearly define risk factors and ascertain possible reasons for the high prevalence observed is encouraged.

\section{Conflict of Interest}

The authors declare that no known conflict of interest exists

\section{Acknowledgements}

The authors acknowledge the support of Management and staff of Adeoyo Maternity Teaching Hospital, Yemetu, Ibadan, Nigeria; St. Mary Specialist Hospital, Eleta, Ibadan, Nigeria and University College Hospital, Ibadan, Nigeria. We thank Dr. MO Adewumi for his unrelenting support and cooperation. We also thank Mr. NA Fashina and Miss GH Adesokan for the samples and all the pregnant women for their consent, cooperation and participation.

\section{REFERENCES}

[1] Kurth, R. and Bannent, N. (eds.) (2010) Retroviruses: Molecular Biology, Genomics and Pathogenesis. Castier Academic Press, United Kingdom.

[2] Quinn, T. C. (2011) Epidemiology of HIV Infection and AIDS in: Goldmann, L. and Schafer, A. I. (eds.) Cell Medicine. 24th Ed. Sanders Elsevier, Philadelphia, PA.

[3] Mushahwar, I. (2007) Human Immunodeficiency Viruses, Molecular Virology. Pathogenesis, Diagnosis and Treatment. Perspectives in Medical Virology 13: 75 - 87.

[4] Oleribe, O. O., Aliyu, S. and Taylor-Robinson, S. D. (2018) is the prevalence of HIV wrongly estimated in Nigeria? Some insights from a 2017 World AIDS day experience from a Nigerian Non-Governmental Organisation. Pan African Medical Journal 29: 119.

[5] Federal Ministry of Health, FMoH [Nigeria] (2014) National HIV Sero-prevalence Sentinel Survey among Pregnant Women Attending Antenatal Clinics in Nigeria, 201 Fact Sheet. Department of Public Health National AIDS/ STI Control Programme, Abuja, Nigeria.
[6] United Nations Programme on HIV and AIDS, UNAIDS (2018) Ending the AIDS Epidemic, Fact Sheet [online]. Retrieved from http://www.unaids.org/sites/default/files/m edia_asset/UNAIDS_Fact Sheet en.pdf, accessed 10th May, 2018.

[7] World Health Organisation (WHO) and United Nations Programme on HIV and AIDS (UNAIDS) (2015) Global AIDS Response Progress Reporting 2015. WHO, Geneva, Switzerland.

[8] National Agency for the Control of AIDS, NACA (2016) Global AIDS Response - Country Progress Report (Nigeria GARPR 2015). NACA, Abuja, Nigeria.

[9] Singh, S., Mahajan, S., Singh, T. and Deepti, S. S. (2018) Socio-demographic and clinical profile of HIV/AIDS patients attending the ART centre of Amritsar, Punjab. International Journal of Community Medicine and Public Health 5 (5): 2059 - 2065.

[10] Kharsanyi, A. B. M. and Karim, Q. A. (2016) HIV Infection and AIDS in Sub-Saharan Africa: Current Status, Challenges and Opportunities. The Open AIDS Journal 10: $34-48$.

[11] Kasonde, G. M., Masaka, M. and Michelo, C. (2018) Trends in HIV prevalence among pregnant women in Lusaka Province, Zambia 1994 - 2008. Journal of Transmitted Diseases and Immunity 2(1): $1-9$.

[12] Anyaka, C., Tinuade, O., Jonah, M., Isichei, M., Anyaka, I. and Isichei, C. (2016) HIV Prevalence amongst Pregnant Women Clients Attending Antenatal Clinic at the Faith Alive Foundation and PMTCT Centre, Jos Plateau State. World Journal of AIDS 6: 59-64.

[13] Awofala, A. A. and Ogundele, O. E. (2018) HIV Epidemiology in Nigeria. Saudi Journal of Biological Sciences 25: 697-703.

[14] Okerentugba P. O., Uchendu S. C. and Okonko I. O. (2015) Prevalence of HIV among pregnant women in Rumubiakani, Port Harcourt, Nigeria. Pub Health Res. 5(2): 58 - 65.

[15] Fouedjio, J. H., Fouelifack, F. Y., Fouelifa, L. D. and Mbu, R. E. (2017) Prevalence and associated factors of HIV infection among pregnant women attending antenatal care at the Yaoundé central hospital. International Journal of Reproduction, Contraception, Obstetrics and Gynaecology 6(7): $2698-2703$.

[16] Aghoghoroma, C. O. and Iliyasu, Z. (2015) HIV Prevalence and Trends among Pregnant Women in Abuja, Nigeria: A 5-Year Analysis. Trop J Obstet Gynaecol 32 (1): 82 - 89.

[17] Eaton, J. W., Rehle, T. M., Jooste, S., Nkambule, R., Kim, A. A., Mahy, M. and Hallet, T. B. (2014) Recent HIV prevalence trends among pregnant women and all women in sub-Saharan Africa: implications for HIV estimates. AIDS 28 (suppl 4): S507 - S514.

[18] Maling S, Todd J, Van Paal L, Grosskurth H, Kinyanda E (2011) HIV-1 seroprevalence and risk factors for HIV infection among first time psychiatric admissions in Uganda. AIDS Care 23: 171 - 178.

[19] Isaac, E., Haruna, M., Samuel, O., Deborah, E., Maikano, M., Sharafudeen, A., Sheik, T. L. and Olatunbosun, S. (2015) The Sero-prevalence of HIV/AIDS among Patients 
Screened from 2008-2013 Attending Federal Neuropsychiatric Hospital Barnawa Kaduna State North West Nigeria. British Microbiology Research Journal 8(3): $507-512$.

[20] United Nations Programme on HIV and AIDS, UNAIDS (2015) AIDS by Numbers 2015. UNAIDS, Geneva, Switzerland.

[21] Sharma, A. and Chauhan, N. (2018) Seroprevalence of HIV among General Population Attending at a Tertiary Care Hospital in Udaipur, Rajasthan, India. Int. J. Curr. Microbiol. App. Sci, (2018) 7(6): 1774 - 1779.

[22] Gill, M.M., Machekano, R., Isarwa, A., Ahimsibwe, A., Oyebanji, O., Akintade, O. L. and Tiam, A. (2015) The Association between HIV Status and Antenatal Care Attendance among Pregnancy Women in Rural Hospitals in Lesotho. Journal of Acquire Immune Deficiency Syndromes, 68: e33-e38.

[23] Agida, E. T., Abu, P., Echikwonye, M., Hwande, T.S., Ayeni, H.A.D. and Swende, T.Z. (2010) HIV Prevalence among Clients Attending Antenatal Clinic at the Federal Medical Centre Makurdi. Nigerian Medical Journal, 19, 295 $-297$.

[24] La'ah, J. G., and Ayiwulu, E. (2010). Socio-Demographic Characteristics of Patients Diagnosed with HIV/AIDS in Nasarawa Eggon. Asian Journal of Medical Sciences 2(3): 114-120.

[25] Okonko I O, Adeniji F O, Okerentugba P O, Anugweje KC (2012) Detection of HIV $1 \& 2$ Antibodies among Intending Blood Donors. Nature and Science 10(5): $1-6$.

[26] Sule W. F., Okonko I. O., Yusuf O. T., Donbraye E., Fadeyi A. and Alli J. A. (2010) HIV-1 and -2 Antibodies among Children in Anyigba, Kogi State, Nigeria. Asian Journal of Medical Sciences 2(4): $170-176$.

[27] Isichie, C., Brown, P., Isichie, M., Njab, J., Oyebode, T. and Okonkwo, P. (2015) High Rate of HIV Prevalence and Associated Risk Factors among Rural Pregnant Women in North Central Nigeria. America Journal of Health Research, 3: $18-23$.

[28] Kajubi P, Green E C, Hudes E S, Kamya M R, Ruark AH (2011) Multiple sexual partnerships among poor urban dwellers in Kampala, Uganda. J Acquir Immune Defic Syndr. 57: 153-156.

[29] Maher D, Waswa L, Karabarinde A, Baisley K. (2011) Concurrent sexual partnerships and associated factors: A cross-sectional population-based survey in a rural community in Africa with a generalised HIV epidemic. BMC Publ Health 11: 651. 